\title{
Optimized Production of Disulfide-Bonded Fungal Effectors in Escherichia coli Using CyDisCo and FunCyDisCo Coexpression Approaches
}

\author{
Daniel S. Yu, ${ }^{1}$ Megan A. Outram, ${ }^{1}$ Emma Crean, ${ }^{1}$ Ashley Smith, ${ }^{1}$ Yi-Chang Sung, ${ }^{1}$ Reynaldi Darma, ${ }^{1}$ \\ Xizhe Sun, ${ }^{1,2}$ Lisong Ma, ${ }^{1}$ David A. Jones, ${ }^{1}$ Peter S. Solomon, ${ }^{1}$ and Simon J. Williams ${ }^{1, \dagger}$ \\ ${ }^{1}$ Research School of Biology, The Australian National University, Canberra, ACT 2601, Australia \\ ${ }^{2}$ Key Laboratory of Hebei Province for Plant Physiology and Molecular Pathology, College of Life Sciences, Hebei Agriculture \\ University, Baoding, China
}

Accepted 20 October 2021.

Effectors are a key part of the arsenal of plant-pathogenic fungi and promote pathogen virulence and disease. Effectors typically lack sequence similarity to proteins with known functional domains and motifs, limiting our ability to predict their functions and understand how they are recognized by plant hosts. As a result, cross-disciplinary approaches involving structural biology and protein biochemistry are often required to decipher and better characterize effector function. These approaches are reliant on high yields of relatively pure protein, which often requires protein production using a heterologous expression system. For some effectors, establishing an efficient production system can be difficult, particularly those that require multiple disulfide bonds to achieve their naturally folded structure. Here, we describe the use of a coexpression system within the heterologous host Escherichia coli, termed CyDisCo (cytoplasmic disulfide bond formation in $E$. coli) to produce disulfide bonded fungal effectors. We demonstrate that $\mathrm{CyDisCo}$ and a naturalized coexpression approach termed FunCyDisCo (Fungi CyDisCo) can significantly improve the production yields of numerous disulfide-bonded effectors from diverse fungal pathogens. The ability to produce large quantities of functional recombinant protein has facilitated functional studies and crystallization of several of these reported fungal effectors. We suggest this approach could be broadly

${ }^{\dagger}$ Corresponding author: S. J. Williams; simon.williams@anu.edu.au

Funding: This work was supported by the Australian Research Council (ARC) through grant DP180102355 to P.S. Solomon and grant DP200100388 to D. A. Jones and S. J. Williams, L. Ma was funded by an ARC Discovery Early Career Researcher Award (DE170101165), and S. J. Williams was funded by an ARC Future Fellowship (FT200100135) and is supported by the Australian National University Future Scheme (35665). E. Crean and A. Smith were recipients of the Australian Institute of Nuclear Science and Engineering (AINSE) Honours Scholarship Program and D. S. Yu holds an AINSE Postgraduate Research Award. This work was also supported by an Australian Academy of Science Thomas Davies Research Grant.

*The $\boldsymbol{e}$-Xtra logo stands for "electronic extra" and indicates there are supplementary materials published online.

The author(s) declare no conflict of interest. distributed under the CC BY-NC-ND 4.0 International license. useful in the investigation of the function and recognition of a broad range of disulfide bond-containing effectors.

Keywords: coexpression system, CyDisCo, disulfide-rich proteins, fungal effectors, protein expression

Fungal pathogen infections are a leading cause of yield losses in many economically important crops. During infection and colonization of their plant host, fungal pathogens utilize small, secreted virulence proteins known as effectors to promote disease (Stergiopoulos and de Wit 2009). Characterized effectors have been implicated in functions that include the targeting and disruption of plant defenses and nutrient acquisition from the host (Selin et al. 2016). Effectors can also be recognized by plant receptors, which activate defense pathways leading to plant immunity (Dodds and Rathjen 2010).

Fungal pathogens utilize effectors during colonization of plant hosts (Oliveira-Garcia and Valent 2015). Understanding how these effectors function is often challenging. Many effectors have low sequence similarity to proteins with known functional domains or motifs, preventing reliable functional predictions based on sequence alone. The most informative effector function studies often require multidiscipline approaches.

We are interested in understanding the structure and function of effectors from multiple plant-pathogenic fungi. Many of these are cysteine-rich effectors, whereby the thiol groups of the cysteine sidechain form intramolecular disulfide bonds (Outram et al. 2021). To study disulfide bond-containing effectors, we have sought to develop tools to enhance protein production in Escherichia coli (Outram et al. 2021; Zhang et al. 2017). To this end, we (and others), have had success using the specialized strain of E. coli, SHuffle (New England Biolabs, Ipswich, MA, U.S.A.) (De la Concepcion et al. 2018; Maqbool et al. 2015; Outram et al. 2021; Zhang et al. 2017). SHuffle is engineered to address unfavorable redox potential in the cytoplasm, through disruption of the glutaredoxin and thioredoxin pathways, and expression of a cytoplasmic version of the disulfide bond isomerase protein DsbC, which normally localizes to the periplasm (Fig. 1A and B). These manipulations have been shown to improve production of correctly folded disulfide-bonded proteins (Lobstein et al. 2012). We have subsequently utilized small solubility tags to further enhance disulfide-rich effector yields in SHuffle (Outram et al. 2021). Despite these advances, the yields obtained for many of our effectors of interest 


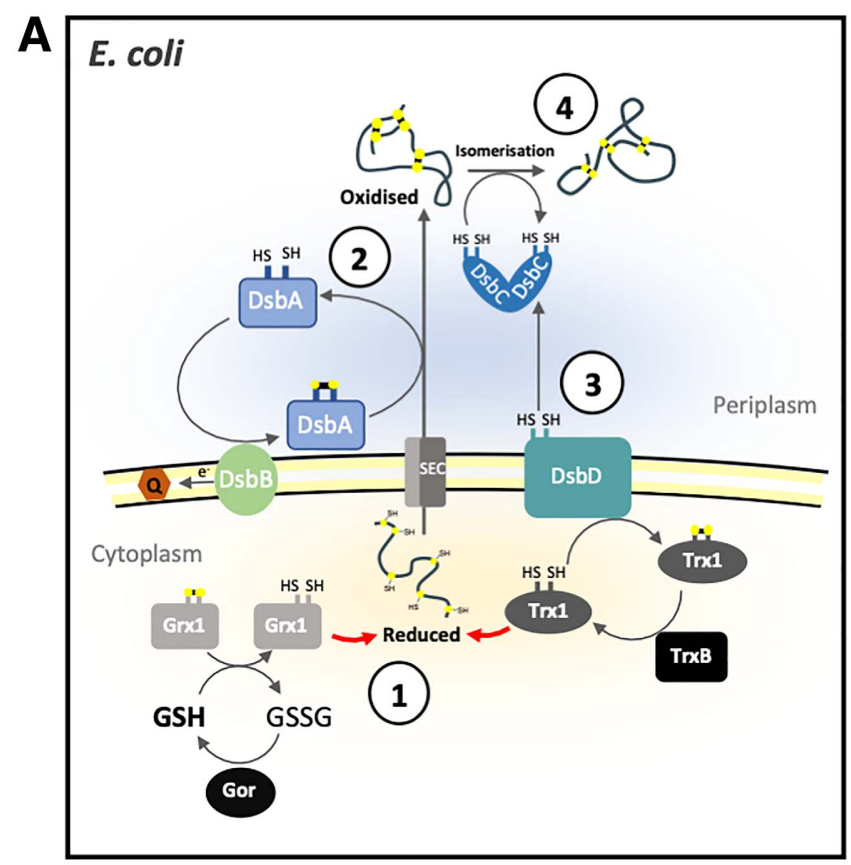

B

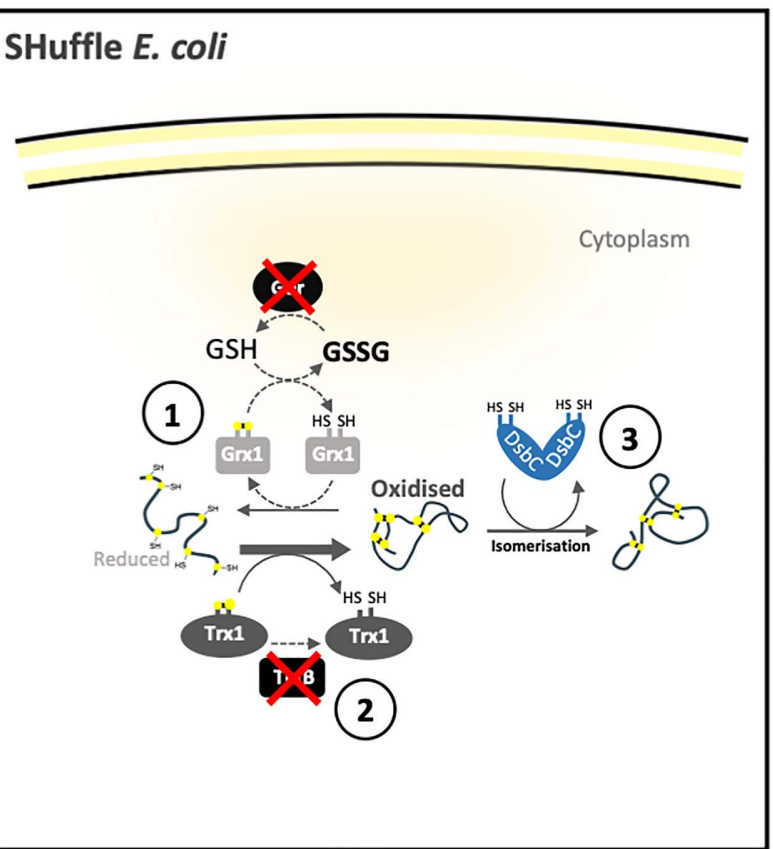

C

SHuffle E. coli with CyDisCo Co-expression

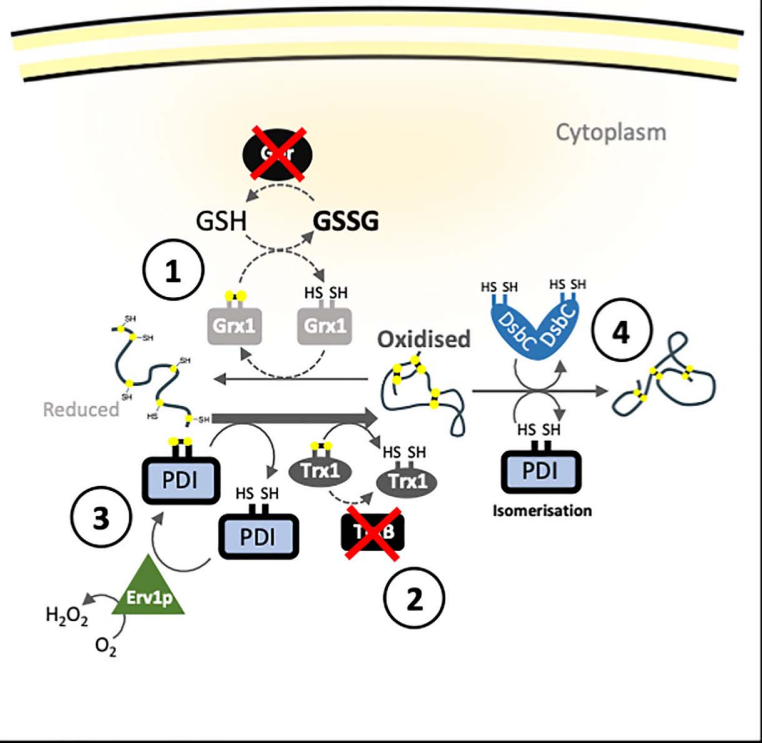

Fig. 1. Disulfide bond formation in Escherichia coli expression systems. A, Expression of proteins in wild-type E. coli. 1) The cytoplasm is a reducing environment. Contributing factors include the high reduced glutathione (GSH):oxidized glutathione (GSSG) ratio maintained by glutathione reductase (Gor). Excess GSH reduces glutaredoxin-1 (Grx1), which can then reduce nascent proteins. Proteins in the cytoplasm can also be reduced by thioredoxin-1 (Trx1), which maintains its reducing power by thioredoxin reductase (TrxB). 2) Newly translated proteins are transported out of the cytoplasm into the periplasm in which they are oxidized by DsbA, which is regenerated by DsbB. Electrons are accepted by ubiquinone (Q) and are carried to the electron transport chain. 3) Incorrectly disulfide-bonded proteins can be isomerized by DsbC. For isomerization to occur, DsbC needs to be in a reduced or hemireduced state, which is maintained by DsbD. The redox state of DsbD is reset by cytoplasmic Trx 1. 4) Reduced DsbC can isomerize the disulfide bonds resulting in correct disulfide-bonding. B, In SHuffle E. coli (Lobstein et al. 2012), the redox state of the cytoplasm is altered to be more oxidizing. 1) The cytoplasm of SHuffle has a lower GSH:GSSG ratio due to a Gor knockout weakening the reduction pathway. 2) TrxB knockout prevents the reduction of Trx 1, which resets the redox state of DsbD. The higher proportion of oxidized Trx 1 strengthens the oxidation pathway and newly translated proteins can be oxidized in the cytoplasm. 3) SHuffle E. coli is engineered to cytoplasmically express DsbC, which can isomerize incorrectly disulfide-bonded proteins in the cytoplasm. C, CyDisCo coexpression in SHuffle E. coli further strengthens the oxidation pathway in the cytoplasm. 1) In SHuffle E. coli, the Gor knockout weakens the reduction pathway and 2) the TrxB knockout strengthens the oxidation pathway. 3) CyDisCo coexpression of protein disulfide isomerase (PDI) readily oxidizes newly translated proteins in the cytoplasm. The redox state of PDI is reset by the sulfhydryl oxidase Erv1p, which generates de novo disulfide bonds by donating electrons on to $\mathrm{O}_{2}$. Erv1p can also oxidize proteins. 4) Incorrectly disulfide-bonded proteins are isomerized by cytoplasmically expressed DsbC from SHuffle and PDI from CyDisCo. 
have remained low and inadequate for structural and biochemical studies.

To address this limitation, we have sought to further improve our production system. The emergence of synthetic biology and the molecular tools that support this discipline have seen an increased interest in coexpression of eukaryotic machinery and chaperones in E. coli to improve recombinant protein production (Zhou et al. 2018). This approach has also been developed to enhance production of disulfide-bonded proteins. Matos et al. (2014) introduced the CyDisCo system (for cytoplasmic disulfide bond formation in $E$. coli), involving coexpression, in $E$. coli, of a disulfide-bonded protein of interest with a yeast mitochondrial sulfhydryl oxidase, Erv1p, and human protein disulfide isomerase (PDI) (Fig. 1C). To date, enhanced production of numerous disulfide-rich human proteins has been reported, including antibodies, human growth factor, and perlecan (Gaciarz et al. 2016; Matos et al. 2014; Sohail et al. 2020). More recently, the CyDisCo system has been used to produce functional recombinant SARS-CoV-2 spike receptor binding domain (Prahlad et al. 2021).

Here, we demonstrate the utility of the CyDisCo coexpression system in combination with SHuffle E. coli to produce disulfiderich fungal effectors. Using this system, seven of eight effector candidates studied were successfully purified with higher yields (ranging from $2 x$ to $29 x$ ) compared with SHuffle alone. We sought to naturalize the system further toward the production of fungal effectors by utilizing a native PDI and sulfhydryl oxidase from Fusarium oxysporum f. sp. lycopersici. The naturalized system, termed FunCyDisCo, outperformed protein production using SHuffle alone and had varied, proteindependent results, compared with $\mathrm{CyDisCo}$. In our hands, the adoption of $\mathrm{CyDisCo} /$ FunCyDisCo has enabled the functional and structural investigation of numerous disulfide-rich effectors that could not otherwise be achieved. We suggest this approach could be broadly useful in the investigation of the function and recognition of a broad range of disulfide-bond containing effectors.

\section{RESULTS}

\section{CyDisCo facilitates the improved production of SIX6 proteins from Fusarium oxysporum.}

We have previously demonstrated that numerous $F$. oxysporum f. sp. lycopersici secreted in xylem (SIX) effectors can be produced using the $E$. coli strain SHuffle in combination with an N-terminal GB1 (protein GB1 domain) solubility tag (Outram et al. 2021). Nevertheless, yields remained relatively low for some effectors of interest, including SIX6 (approximately $0.3 \mathrm{mg}$ per liter of culture), and made structural studies difficult and laborious (Outram et al. 2021). To address this limitation, we employed CyDisCo, which involves coexpression of a sulfhydryl oxidase and PDI with the effector of interest in SHuffle E. coli (Fig. 1C). To understand the effectiveness of this approach, we performed side-by-side expression and purification of N-terminal 6xHis-GB1 tagged SIX6 (lacking the signal peptide) in SHuffle alone or in SHuffle with CyDisCo (Fig. 2A). GB1-SIX6 produced in SHuffle alone was highly expressed; however, most of the protein was insoluble. The total amount of SIX6, when coexpressed with CyDisCo was lower compared with SHuffle alone; however the total protein and soluble fraction (clarified lysate) were indistinguishable, suggesting improved solubility. GB1-SIX6 expressed with and without CyDisCo was subsequently purified from the soluble fractions, using nickel affinity chromatography (Fig. 2B). The protein yields obtained for GB1-SIX6 with CyDisCo were greater than GB1-SIX6 produced in SHuffle alone and of higher purity, as determined by sodium dodecyl sulfate-polyacrylamide gel electrophoresis (SDS-PAGE) analysis (Fig. 2B). We removed the GB1 fusion partner from SIX6 using 3C protease and used an additional nickel purification step to remove the GB1-tag, uncleaved protein, and the $3 \mathrm{C}$ protease, prior to further purification by size exclusion chromatography (SEC). More monodispersed SIX6 protein was obtained when expressed with CyDisCo than with SHuffle alone (Fig. 2C). The final yields were $1.5 \mathrm{mg}$ per liter for SIX6 coexpressed with CyDisCo, compared with $0.4 \mathrm{mg}$ per liter when expressed alone in this experiment. We consistently obtaining higher final yields (ranging from 0.9 to $2.2 \mathrm{mg}$ per liter) for SIX6 coexpressed with CyDisCo compared with SIX6 expressed in SHuffle alone (from 0.2 to $0.5 \mathrm{mg}$ per liter) in three independent replicates of this experiment, highlighting the robustness and reproducibility of the coexpression approach. The quality of the purified SIX6 was analyzed using intact protein mass spectrometry (MS) and circular dichroism (CD) spectroscopy, which revealed that the protein is disulfide-bonded (four disulfides formed) and contains secondary structural elements dominated by $\beta$-sheets (Supplementary Figs. S1 and S2).

Several homologs of SIX6 exist in different formae speciales of $F$. oxysporum, ranging from 60 to $92 \%$ similarity to SIX6 from $F$. oxysporum f. sp. lycopersici. Notably, all homologs of SIX6 contain conserved cysteine residues (Fig. 2D). To validate the effectiveness of the CyDisCo system for improving protein yields of SIX6, homologs of SIX6 from $F$. oxysporum $\mathrm{f}$. sp. cubense and $F$. oxysporum f. sp. vasinfectum were expressed with and without $\mathrm{CyDisCo}$ and were subsequently purified (Fig. 2E and F). The coexpression of CyDisCo improved the soluble protein yield by approximately fivefold for FocSIX6, from 0.6 to $3.1 \mathrm{mg}$ per liter, and about fivefold for FovSIX6, from 0.04 to $0.2 \mathrm{mg}$ per liter, consistent with the results for FolSIX6. Collectively, these data suggest that CyDisCo promotes improved disulfide-bond formation to boost yields of soluble correctly folded SIX6 in E. coli.

\section{CyDisCo facilitates the improved production of an expanded set of disulfide-rich fungal effectors.}

Based on the success observed for SIX6, we wanted to test the utility of the CyDisCo system to produce different disulfiderich fungal effectors. The effectors chosen include SIX1 (Avr3) and SIX4 (Avr1) from F. oxysporum f. sp. lycopersici, SnTox1 and SnTox3 from Parastagonospora nodorum, and NIP2.1 from Rhynchosporium commune (Supplementary Table S1). Most of these effectors could only be produced in low yields from SHuffle $E$. coli, despite the addition of fusion partners (Outram et al. 2021). SIX1, SIX4, and SnTox3 were expressed with an N-terminal 6xHisGB1 tag, but GB1 was not included for SnTox1 and NIP2.1 as the tag was a similar size to the proteins of interest, leading to complications during downstream analysis. Proteins expressed in SHuffle E. coli alone or with CyDisCo were expressed and purified (side-by-side), using the same approach described for SIX6 (details below), and the final monodispersed SEC elution fractions were compared (Fig. 3). In most cases, we observed an increase in final yields associated with coexpression with CyDisCo, with an approximately 29-fold improvement for SIX1, resulting in a yield of $4.3 \mathrm{mg}$ per liter, am approximately sixfold improvement for SIX4, with a final yield of $2.4 \mathrm{mg}$ per liter, an approximately threefold improvement for SnTox1, with a final yield of $1.5 \mathrm{mg}$ per liter, and an approximately 2.5 -fold improvement for NIP2.1, with a final yield of $0.15 \mathrm{mg}$ per liter. SnTox3 was the only protein that did not show any obvious improvement in yield when coexpressed with CyDisCo. Collectively, this demonstrates a general effectiveness of the CyDisCo coexpression system in improving the yield of multiple disulfide-rich effectors across different fungal species, with the degree of improvement being protein-specific. 
A modified fungal-specific CyDisCo system for improved soluble expression of disulfide-rich fungal effectors.

The Erv1p and human PDI pair of CyDisCo was previously reported to be the most effective at increasing protein yield
(Gaciarz et al. 2016). However, this system has been used predominantly to enhance the production of disulfide-rich human proteins such as antibodies, human growth factor, and perlecan (Gaciarz et al. 2016; Matos et al. 2014; Sohail et al. 2020).

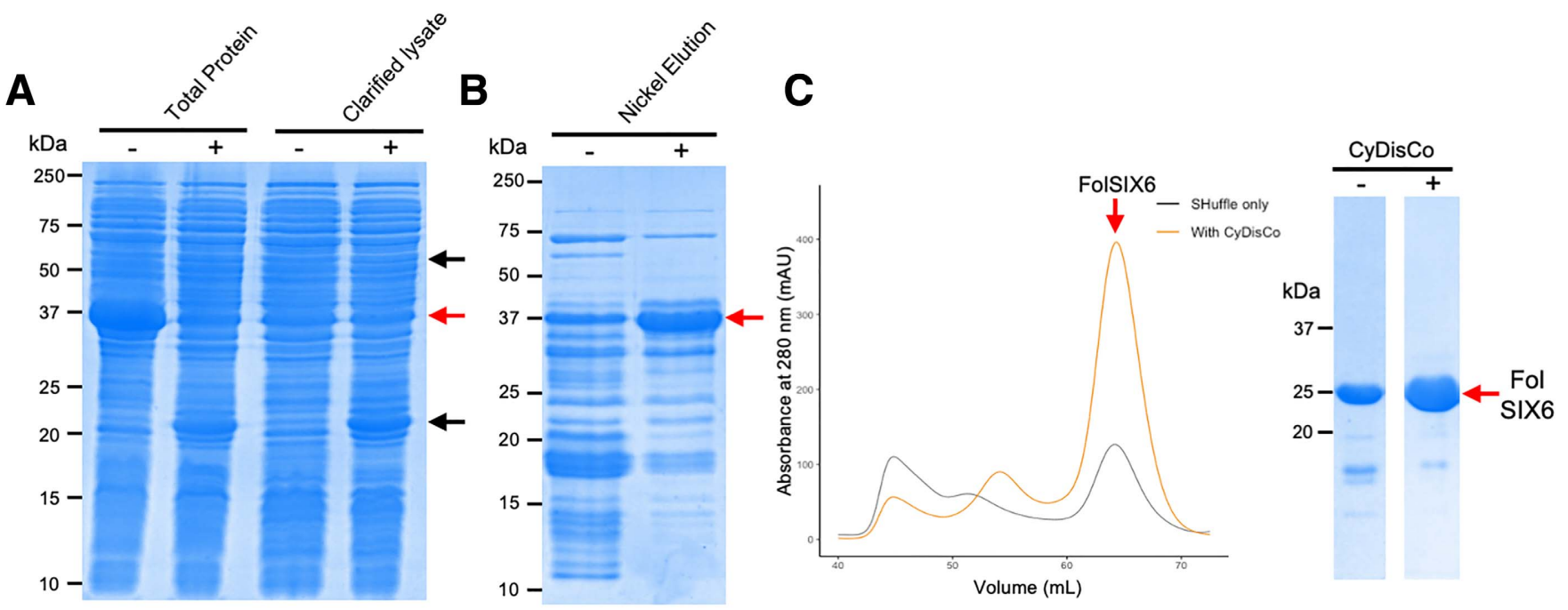

Signal peptide

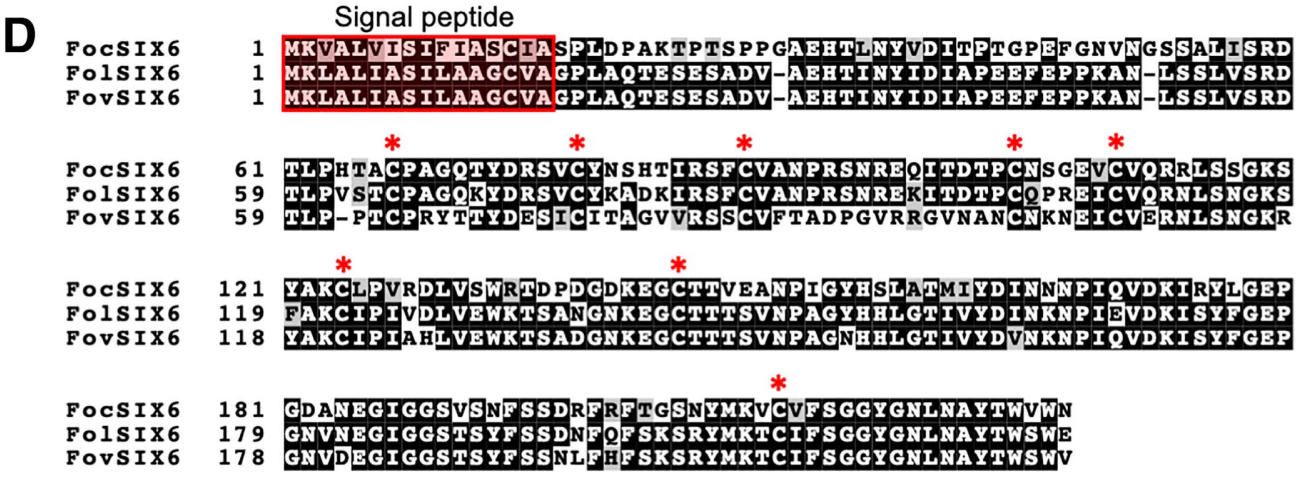

$\mathbf{E}$

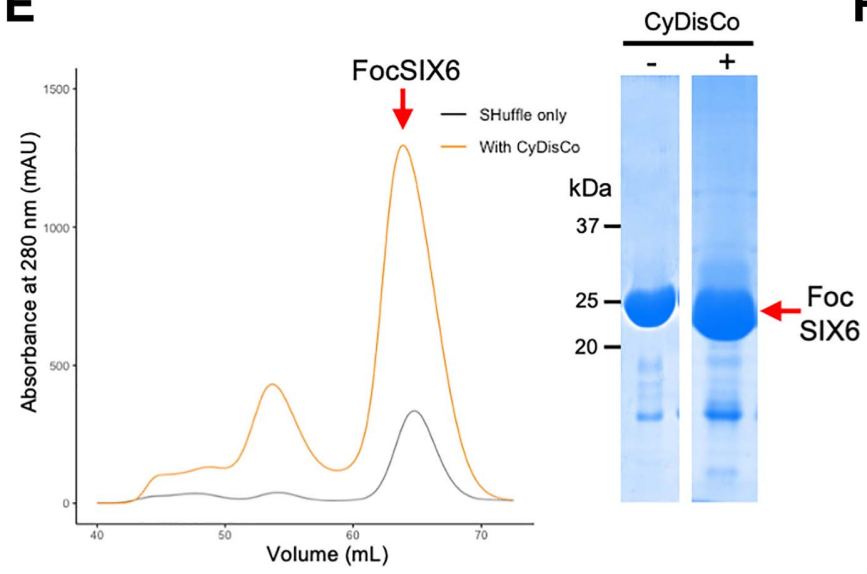

$\mathbf{F}$

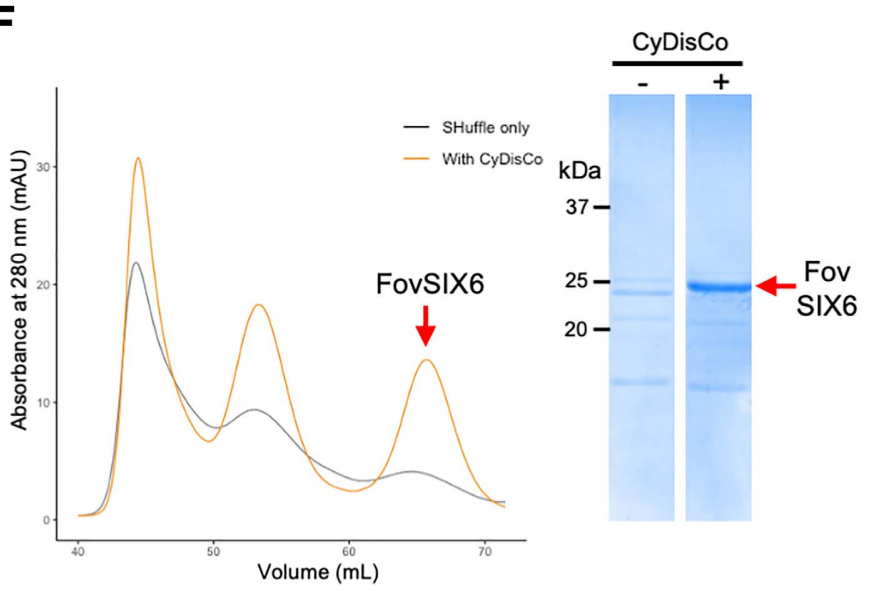

Fig. 2. Recombinant SIX6 protein can be produced in SHuffle Escherichia coli alone but yields are higher when coexpressed with CyDisCo. Coomassiestained sodium dodecyl sulfate-polyacrylamide gel electrophoresis (SDS-PAGE) gel showing A, total and soluble (clarified lysate) proteins and B, proteins captured by immobilized metal affinity chromatography from SHuffle E. coli expressing 6xHisGB1-FolSIX6 with (+) or without (-) CyDisCo coexpression. The red arrows point to 6xHisGB1-FolSIX6 (approximately $37 \mathrm{kDa}$ ), and black arrows indicate the protein disulfide isomerase (approximately $55 \mathrm{kDa}$ ) and Erv1p (approximately $21 \mathrm{kDa}$ ). C, The graph on the left shows size-exclusion chromatograms of FolSIX6 protein produced by SHuffle E. coli, following cleavage by $3 \mathrm{C}$ protease to remove the N-terminal 6xHisGB1 fusion. FolSIX6 produced in SHuffle with CyDisCo is shown in orange and FolSIX6 in SHuffle alone is shown in black. The red arrow points to the peak corresponding to FolSIX6. On the right, Coomassie-stained SDS-PAGE gel showing equal volume loading of FolSIX6 protein (indicated by red arrow) expressed with (+) or without (-) CyDisCo corresponding to the protein peak from size-exclusion chromatograms. D, Sequence alignment of FolSIX6 and two SIX6 homologs from Fusarium oxysporum f. sp. cubense (Foc) and F. oxysporum f. sp. vasinfectum (Fov). The signal peptide is highlighted in red, as determined by SignalP (Almagro Armenteros et al. 2019). Conserved cysteine residues are marked with a red asterisk. E, Size-exclusion chromatogram and SDS-PAGE analysis for FocSIX6 and F, FovSIX6 produced with (orange trace) or without (black trace) CyDisCo coexpression, as presented in C. 
Recently, a modified CyDisCo system was utilized to produce disulfide-rich conotoxins from cone snails, whereby an additional conotoxin-specific PDI from Conus geographus was included with the CyDisCo components (Nielsen et al. 2019).

We have shown that CyDisCo benefits the production of numerous disulfide-rich fungal effectors. Despite this advance, the yield for some effectors, such as FovSIX6, remained low $(0.2 \mathrm{mg}$ per liter) and we wanted to investigate whether the CyDisCo system could be modified and improved to benefit the production of recalcitrant disulfide-rich fungal effectors.
We substituted the human PDI with a PDI from $F$. oxysporum f. sp. lycopersici, as the amino acid sequence of human PDI is substantially divergent from fungal PDIs (Supplementary Fig. S3A). We also selected a sulfhydryl oxidase (Erv2) that localizes in the endoplasmic reticulum (ER) of fungi to coexpress with PDI in place of ERV1p. Erv2 is a fungal-specific membrane-bound sulfhydryl oxidase that catalyzes disulfide bonds de novo within the ER (Sevier and Kaiser 2006; Sevier et al. 2001). When overproduced in yeast, Erv2 forms mixed disulfide bonds with yeast PDI, suggesting a transient association between
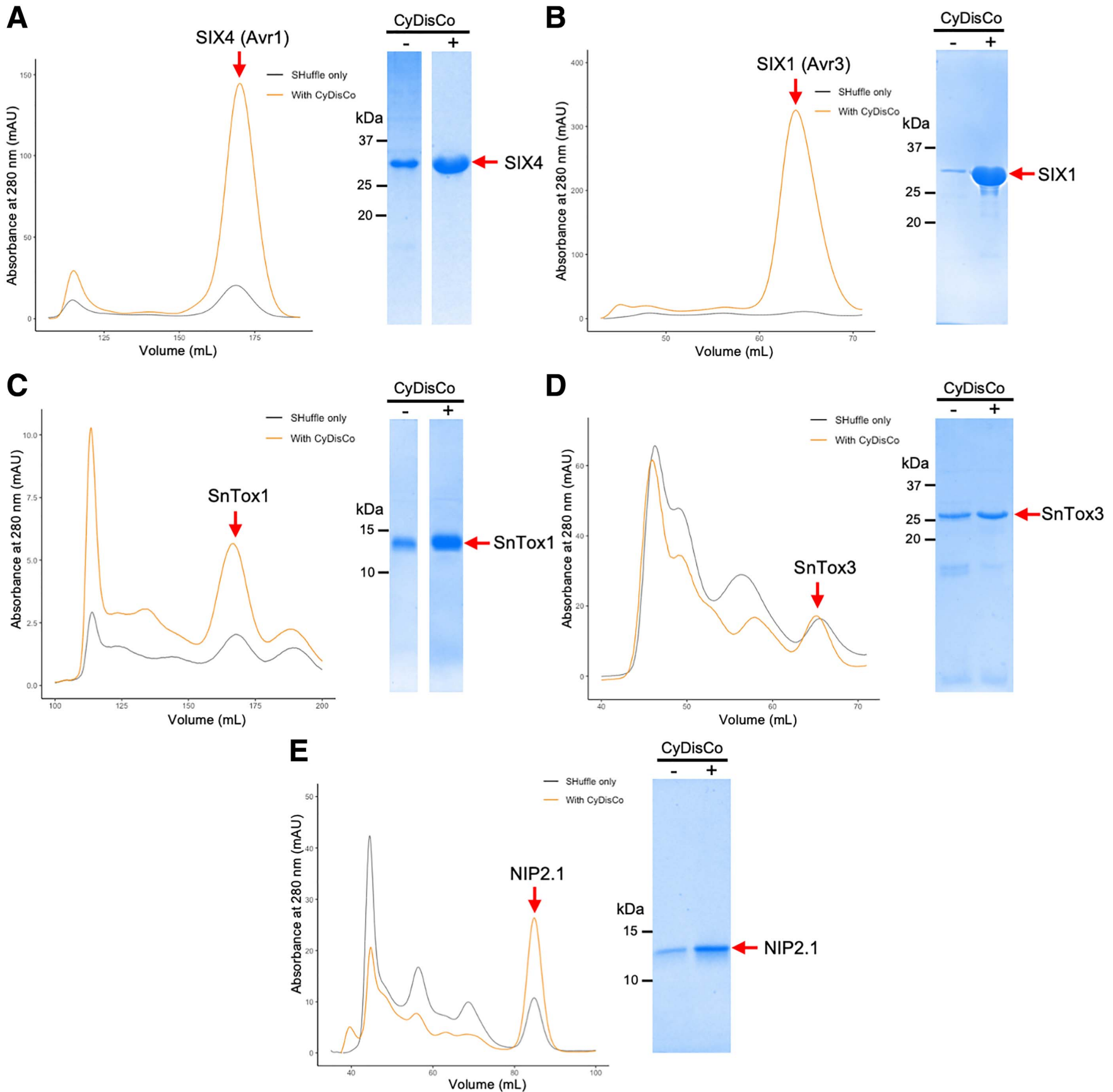

Fig. 3. CyDisCo coexpression in SHuffle Escherichia coli can be used effectively for the production of various disulfide-bonded fungal effector proteins. Graphs on the left present size-exclusion chromatograms of various effectors produced in SHuffle E. coli, following cleavage by $3 \mathrm{C}$ protease to remove their N-terminal fusions, with CyDisCo coexpression (orange) or without (black). The red arrows indicate the peaks corresponding to the effectors of interest. On the right of the graphs, Coomassie-stained sodium dodecyl sulfate-polyacrylamide gel electrophoresis gels showing equal volume loading of effectors of interest (indicated by red arrow) expressed with (+) or without (-) CyDisCo corresponding to the protein peak from size-exclusion chromatograms. Chosen effectors are A, SIX4 (Avr1) and B, SIX1 (Avr3) from Fusarium oxysporum f. sp. lycopersici, C, SnTox1 and D, SnTox3 from Parastagonospora nodorum, and E, NIP2.1 from Rhynchosporium commune. 
the two proteins (Sevier et al. 2001). We selected Erv2 for two reasons. First, PDIs localize to the ER and would not interact with Erv1p-like sulfhydryl oxidases, which localize in the mitochondria (Ellgaard and Ruddock 2005; Lange et al. 2001). Second, the presence of signal peptides in fungal effectors indicate that they are trafficked through the ER and Golgi secretory pathway (Petre and Kamoun 2014).

BlastP searches of the $F$. oxysporum $\mathrm{f}$. sp. lycopersici genome (Ma et al. 2010), using yeast PDI and Erv2 as queries, indicated that four putative PDI proteins and two Erv2-like proteins were present in F. oxysporum f. sp. lycopersici (Supplementary Fig. S3A and B). To select the most appropriate proteins for coexpression studies in $E$. coli, we made use of RNA-seq data from $F$. oxysporum f. sp. lycopersici infections of tomato (Fig. 4A). This demonstrated that FOXG_00140 (FolPDI) and FOXG_09255 (FolErv2) were upregulated during infection, and these proteins were subsequently selected for expression trials (Fig. 4B).

To assess whether we could improve the CyDisCo system for production of disulfide-rich fungal effectors in E. coli, F. oxysporum effectors FolSIX6, FovSIX6, and SIX1, with an N-terminal 6xHisGB1 tag, and NIP2.1 from $R$. commune, with an N-terminal 6xHis tag in SHuffle E. coli, were coexpressed with either $\mathrm{CyDisCo}$ or the modified fungal-specific CyDisCo (FunCyDisCo) containing FolErv2 lacking the N-terminal transmembrane domain and FolPDI lacking the signal peptide (Fig. 4B) and purified them (side-by-side) using the same approaches detailed above. To confirm CyDisCo/FunCyDisCo components were expressed in a soluble form, total and clarified lysates were analyzed by SDS-PAGE (Fig. 4C). For FolSIX6, the coexpression of CyDisCo or FunCyDisCo were equally effective, each resulting in a yield of approximately $2 \mathrm{mg}$ per liter of culture, a fivefold increase compared with SHuffle alone (Fig. 4D). FovSIX6 coexpressed with FunCyDisCo resulted in a yield of approximately $0.6 \mathrm{mg}$ per liter of culture, a threefold improvement in yield compared with coexpression with CyDisCo and 15-fold improvement compared with SHuffle alone (Fig. 4E). For SIX1, coexpression with FunCyDisCo resulted in a 13-fold improvement in yield compared with SHuffle alone and a twofold decrease in protein yield when compared with CyDisCo (Fig. 4F). NIP2.1 coexpressed with FunCyDisCo resulted in a yield of approximately $0.05 \mathrm{mg}$ per liter, which was similar to the yields obtained from SHuffle alone but an approximately threefold decrease compared with CyDisCo (Fig. 4G). Collectively, these results suggest the use of CyDisCo or FunCyDisCo coexpression systems can both improve yields of disulfiderich effectors compared with SHuffle $E$. coli alone; however, the choice of which system works best is ultimately proteinspecific.

\section{Coexpression of disulfide-rich fungal effectors in nonredox mutant $E$. coli strains.}

We have shown the CyDisCo and FunCyDisCo coexpression systems are effective at improving yields for numerous disulfide-rich fungal effectors produced in SHuffle E. coli compared with SHuffle alone. In a previous report, the CyDisCo system could be used to produce disulfide-bonded antibody fragments in different $E$. coli strains (Gaciarz et al. 2016). This could allow greater flexibility in the choice of $E$. coli background for the expression of disulfide-rich effectors, which might be advantageous for different applications.

We therefore investigated if improvements to the yield of disulfide-rich fungal effectors can be made using the CyDisCo and FunCyDisCo systems expressed in nonredox mutant strains such as BL21(DE3). FolSIX6 lacking the signal peptide with an N-terminal 6xHisGB1 tag was expressed in BL21(DE3) by itself or coexpressed with CyDisCo or FunCyDisCo systems and was purified simultaneously from both, using nickel affinity chromatography. However, we were unable to produce high quantities of FolSIX6 with either coexpression system in BL21(DE3). We were also unable to confirm the soluble production of CyDisCo/FunCyDisCo components (Supplementary Fig. S4). Collectively, in our hands, the CyDisCo and modified FunCyDisCo systems were not transferable into BL21(DE3) E. coli.

\section{Recombinant SIX4 (Avr1) causes cell death in $I$-containing tomato cultivars.}

The adoption of the CyDisCo/FunCyDisCo system has facilitated the structural elucidation of numerous fungal effectors in our lab (structures to be presented elsewhere). Here, however, we present data to show that these high-quality, high-purity proteins have applications outside of structural studies. Previously, we used a protein-mediated phenotyping approach to study the necrotrophic effector SnTox1 and SnTox3 in wheat (Outram et al. 2021; Sung et al. 2021; Zhang et al. 2017). Here, we were interested in determining whether the effectors produced using our enhanced production system could be used to study effector recognition. We demonstrated that purified SIX4 (Avr1) protein infiltrated into cotyledons caused cell death in a tomato cultivar that contained the I-resistance gene (M82). Importantly, cell death was not observed when the same protein was infiltrated into a tomato cultivar lacking $I$ (Moneymaker) (Fig. 5). This demonstrates the capacity for E. coli-produced SIX4 (Avr1) to be recognized by the I resistance protein in the native tomato system.

\section{DISCUSSION}

Here, we demonstrate that the CyDisCo coexpression strategy has the capacity to significantly increase the yield of functional disulfide-rich effectors when produced in SHuffle E. coli. Of the eight effectors we tried, all could be expressed and purified using CyDisCo and seven displayed improved yields and purity compared with SHuffle alone. Our tailored FunCyDisCo outperformed SHuffle alone for the three $F$. oxysporum effectors studied but showed effector-specific differences compared with CyDisCo.

The basis of the CyDisCo coexpression approach is to mimic (albeit loosely) eukaryotic secretory pathways within a prokaryotic host. PDI and sulfhydryl oxidase proteins are known to function together to assist protein folding through disulfide-bond formation and correct pairing of disulfide bonds (Sevier 2010). There is some evidence that these proteins are also important in pathogenic fungi. For example, PDI1 from Ustilago maydis is crucial for the correct folding of a pool of secreted disulfide-rich proteins important for virulence (Marín-Menguiano et al. 2019). We attempted to further tailor this system with the introduction of FunCyDisCo coexpression, using $F$. oxysporum f. sp. lycopersici PDI and Erv2 isoforms identified in RNA-seq data from $F$. oxysporum f. sp. lycopersici-infected tomato. Our data for FunCyDisCo showed mixed success compared with CyDisCo. One potential reason for this variation is isoform specificity. In Saccharomyces cerevisiae, there are more than five PDIlike proteins and two sulfhydryl oxidases localized to the ER, each preferentially aiding the disulfide-bond formation of different proteins (Frand and Kaiser 1999; Nørgaard et al. 2001; Sevier and Kaiser 2006). In F. oxysporum f. sp. lycopersici, four PDI and two Erv2-like homologs can be identified. While RNA-seq data from host infection were used to guide our selection, it is plausible other homologs or combinations could result in better effector yields.

Further improvements may also be derived from the introduction of additional accessory proteins and chaperones that are not specifically involved in disulfide bond formation. For 
example, Lhs1, an HSP70 chaperone from Magnaporthe oryzae, is crucial for the proper processing of secreted proteins, with Lhs1 knockouts exhibiting lower levels of secreted effector proteins and severely reduced pathogenicity (Yi et al. 2009). Other systems involving coexpression of accessory proteins and chaperones have been successfully utilized to express complex proteins in E. coli, such as RuBisCo. The simultaneous coexpression of a plant chaperonin and four assembly factors has been reported

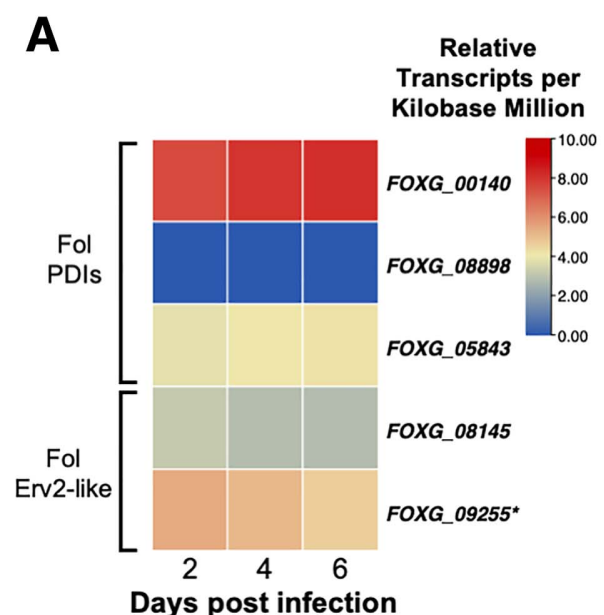

B Signal peptide $21 \mathrm{cc}$

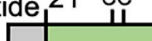

Cytosolic
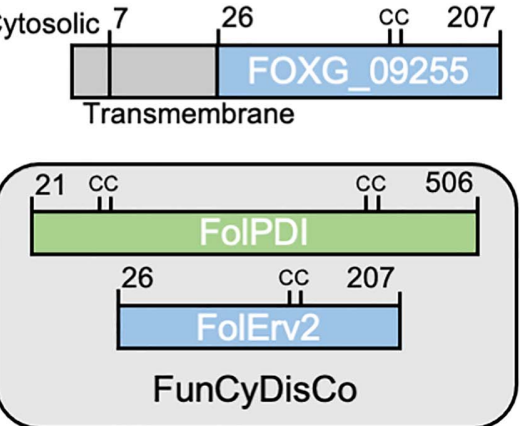

C

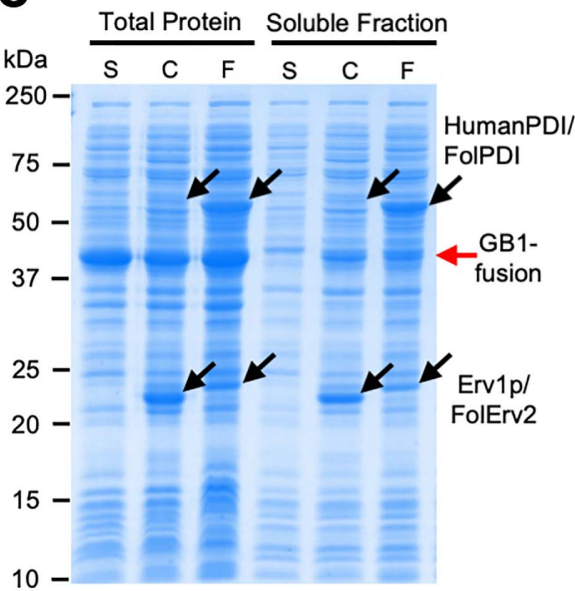

D

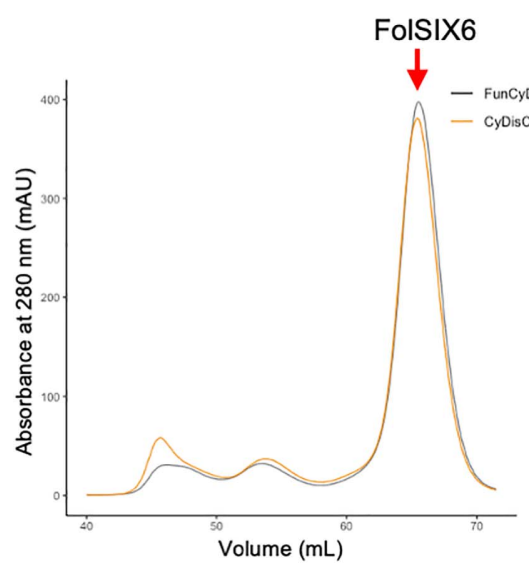

$\mathbf{F}$

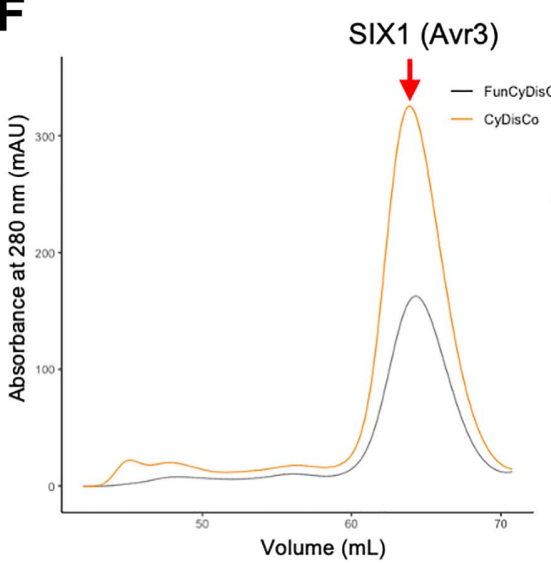

$\mathbf{E}$
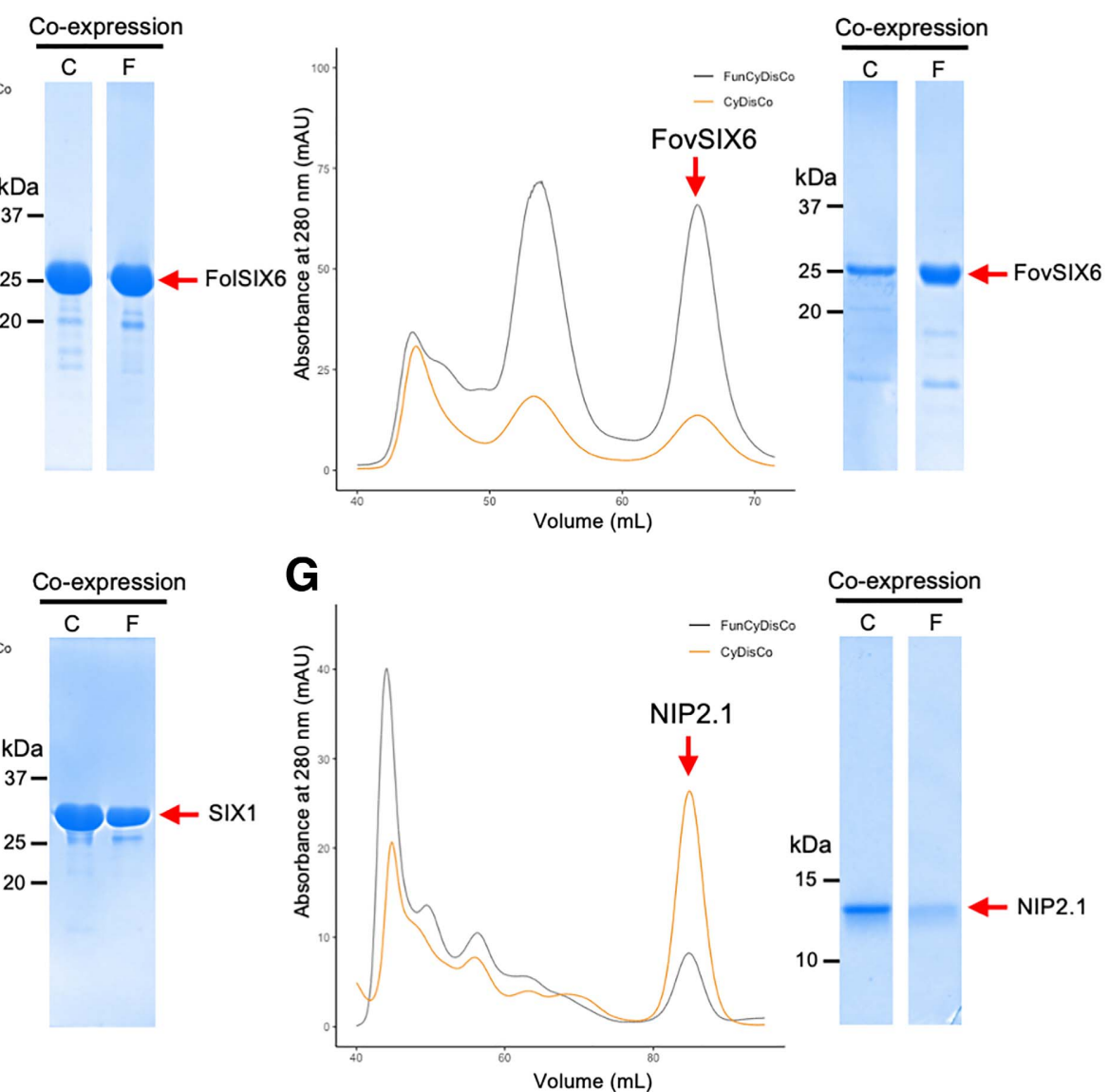

Fig. 4. A fungal-specific CyDisCo system (FunCyDisCo) further improves yields of some effectors. A, RNA-seq analysis of Fusarium oxysporum f. sp. lycopersici protein disulfide isomerases (PDIs) and sulfhydryl oxidases (Erv2-like) during $F$. oxysporum $\mathrm{f}$. sp. lycopersici infection of tomato. Transcripts of $F$. oxysporum f. sp. lycopersici PDIs (FOXG_00140, FOXG_08898, FOXG_05843) and sulfhydryl oxidases (FOXG_08145, FOXG_09255), measured at 2, 4, and 6 days postinfection, are shown. Relative scale represents transcripts per kilobase million (TPM) with values ranging from 0 to 281 TPM. B, Schematic of selected components of FunCyDisCo (top panel) and domains that are expressed (bottom panel). C, Representation of total protein and soluble fractions following expression of a GB1-fusion-effector of interest with the CyDisCo (C) or FunCyDisCo (F) coexpression systems or SHuffle alone (S). Black arrows indicate overexpression of PDI or sulfhydryl oxidase components. D, Size-exclusion chromatogram (left) and sodium dodecyl sulfatepolyacrylamide gel electrophoresis (SDS-PAGE) analysis (right) of the Fusarium oxysporum effectors FolSIX6, E, FovSIX6, F, SIX1(Avr3), and G, Rhynchosporium commune effector NIP2.1 recombinant proteins coexpressed with CyDisCo (C) or FunCyDisCo (F). Red arrows indicate the protein of interest peak on the size-exclusion chromatogram and band on SDS-PAGE gel. Note: FOXG_09255 was incorrectly annotated. The FOXG_09255 sequence has been corrected based on RNA-seq data and can be found in Supplementary Table S2. 
to produce about 12-fold higher yields of functional RuBisCo (Wilson et al. 2019). The incorporation of general accessory proteins and chaperones that aid the oxidative pathway for disulfide-bond formation may tailor the coexpression system for a given protein. However, due to the complexity of different oxidation pathways, pinpointing which proteins to coexpress with a given disulfide-rich effector is difficult.

Eukaryotic proteins produced in a prokaryotic system often end up in inclusion bodies, due to the lack of folding machinery and a rapid rate of protein synthesis preventing correct protein folding (Widmann and Christen 2000). Incorporation of eukaryotic components for coexpression in E. coli to mimic eukaryotic disulfide-bond formation raises the question Why not use a eukaryotic system directly? Eukaryotic expression systems like yeast have been used successfully to express soluble disulfiderich effectors in quantities necessary for structural characterization, such as AvrLm4-7 from Leptosphaeria maculans and Ecp6 from Cladosporium fulvum (Blondeau et al. 2015; SánchezVallet et al. 2013). However, some disulfide-rich proteins were produced in lower quantities when expressed in yeast compared with E. coli, such as SnTox1, SnTox3, and ToxB (Liu et al. 2009, 2012; Outram et al. 2021; See et al. 2019). This demonstrates that choosing expression systems for producing disulfide-rich effectors is not a 'one size fits all' approach.
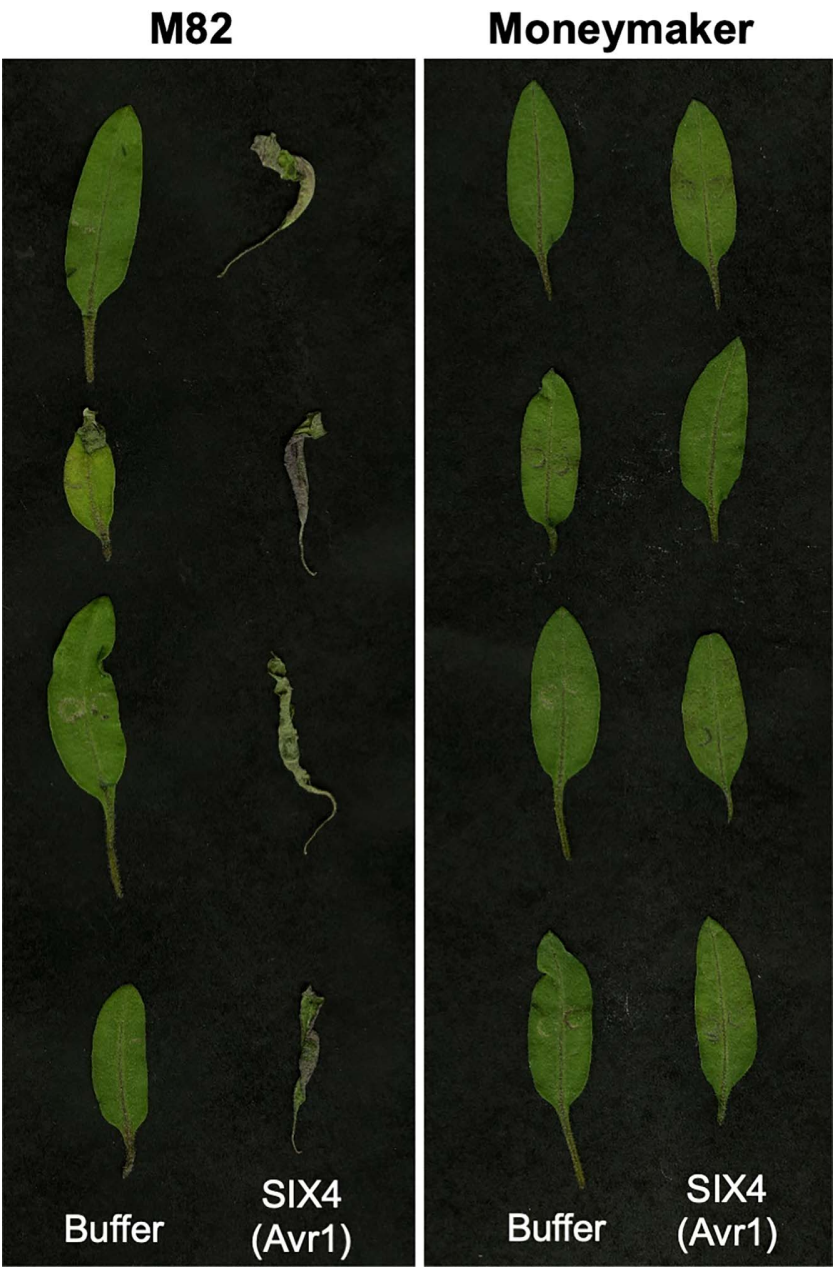

Fig. 5. Escherichia coli-produced SIX4 (Avr1) causes cell death when infiltrated into tomato cultivars containing the resistance gene I. SIX4 (Avr1) (at a concentration of $100 \mu \mathrm{g} / \mathrm{ml}$ ) and a buffer control were syringe-infiltrated into 10-day-old tomato cotyledons from cultivars M82 (containing $I$ ) and Moneymaker (lacking I). Cotyledons were harvested and imaged 4 days postinfiltration.
Therefore, multiple expression systems and strategies should be considered in the early stages of recombinant effector protein production.

With prokaryotic expression systems being cheap and accessible to many laboratories, we believe our combined strategy of the SHuffle E. coli strain, GB1 solubility tag, and CyDisCo or FunCyDisCo coexpression systems would assist the characterization of disulfide-rich effectors from a broad range of plant pathogens.

\section{MATERIALS AND METHODS}

\section{Vectors and gene constructs.}

Fungal effector gene DNA sequences were codon-optimized for expression in E. coli and were synthesized by Integrated DNA Technologies (IDT; Coralville, IA, U.S.A.) (Supplementary Table S2). All genes were cloned into the modified, Golden Gate-compatible, pOPIN expression vector (Bentham et al. 2021). The final expression constructs contained either an N-terminal 6xHis-tag or 6xHis-GB1-tag followed by a 3C protease recognition site. The Golden Gate digestion and ligation reactions and cycling were carried out as described by Iverson et al. (2016).

DNA sequences that encode the yeast Erv1p and human PDI (CyDisCo) and F. oxysporum f. sp. lycopersici Erv2 and F. oxysporum f. sp. lycopersici PDI (FunCyDisCo) were codonoptimized, using the tool provided by IDT, and were synthesized by Twist Bioscience (San Francisco, CA, U.S.A.) (Supplementary Table S2). The yeast Erv1p and human PDI pair and the $F$. oxysporum f. sp. lycopersici Erv2 and $F$. oxysporum f. sp. lycopersici PDI pair were cloned into a modified Golden Gate-compatible pACYC184 vector (GenBank accession number OK442624) using the EcoFlex Kit (Moore et al. 2016), which was a gift from P. Freemont (Addgene kit 1000000080). The Golden Gate digestion and ligation reactions and cycling were carried out as described by the kit (Moore et al. 2016). All plasmid constructs were sequence-verified. The FunCyDisCo and $\mathrm{CyDisCo}$ plasmids were deposited with Addgene (Addgene numbers 176404 and 176405).

\section{Protein expression and purification.}

Sequence-verified effector constructs (approximately $100 \mathrm{ng}$ of plasmid DNA) were chemically transformed into SHuffle T7 Express C3029 (New England Biolabs [NEB], Ipswich, MA, U.S.A.) or BL21(DE3) C2527 competent E. coli (NEB) using the heat-shock protocol provided by the manufacturer and the transformants grown on Luria-Bertani (LB) agar plates supplemented with ampicillin $(100 \mu \mathrm{g} / \mathrm{ml})$ at $37^{\circ} \mathrm{C}$ for $16 \mathrm{~h}$. For $\mathrm{CyDisCo} /$ FunCyDisCo coexpression, the effector of interest and $\mathrm{CyDisCo} /$ FunCyDisCo constructs (approximately $100 \mathrm{ng}$ of plasmid DNA) were transformed simultaneously, and the transformants grown on LB agar plates were supplemented with ampicillin $(100 \mu \mathrm{g} / \mathrm{ml})$ and chloramphenicol $(35 \mu \mathrm{g} / \mathrm{ml})$ at $37^{\circ} \mathrm{C}$ for $16 \mathrm{~h}$. Colonies were used to inoculate LB media supplemented with required antibiotics and were grown overnight at $37^{\circ} \mathrm{C}$ (BL21(DE3) or $30^{\circ} \mathrm{C}$ (SHuffle) with shaking at $220 \mathrm{rpm}$. These small-scale overnight cultures were used to inoculate 1 liter of Teriffic Broth media ( $24 \mathrm{~g}$ per liter yeast extract, $12 \mathrm{~g}$ per liter tryptone, $0.5 \%$ [vol/vol] glycerol, $0.017 \mathrm{M} \mathrm{KH}_{2} \mathrm{PO}_{4}$, $0.072 \mathrm{M} \mathrm{K}_{2} \mathrm{HPO}_{4}$ ) in a 2-liter baffled flask supplemented with required antibiotics and $200 \mu \mathrm{l}$ of Antifoam 204 (Sigma-Aldrich Inc., St. Louis). Large-scale cultures were incubated at $37^{\circ} \mathrm{C}$ [BL21(DE3)] or $30^{\circ} \mathrm{C}$ (SHuffle) with shaking at $220 \mathrm{rpm}$. Cultures were induced with a final concentration of $200 \mu \mathrm{M}$ isopropyl $\beta$-D-1-thiogalactopyranoside once an optical density at $600 \mathrm{~nm}$ of 0.6 was reached and were incubated at $16^{\circ} \mathrm{C}$ with shaking at $220 \mathrm{rpm}$ for a further $16 \mathrm{~h}$. Cells were harvested by 
centrifugation at $4,000 \times g$ for $10 \mathrm{~min}$ at $4{ }^{\circ} \mathrm{C}$. Pellets were resuspended in $50 \mathrm{mM}$ HEPES, $\mathrm{pH} 8.0,300 \mathrm{mM} \mathrm{NaCl}, 10 \%$ (vol/vol) glycerol, $1 \mathrm{mM}$ phenylmethylsulfonyl fluoride and were lysed by sonication, using an amplitude of $40 \%$ (10 s on and $20 \mathrm{~s}$ off). The lysed cells were centrifuged at $20,000 \times g$ for $40 \mathrm{~min}$ to clarify the lysate. The protein of interest was purified further by immobilized metal affinity chromatography (IMAC), using a 5-ml HisTrap FF crude nickel column (Cytiva, Marlborough, MA, U.S.A.). The column was washed, using a buffer containing $50 \mathrm{mM}$ HEPES, pH 8.0, $300 \mathrm{mM} \mathrm{NaCl}, 30 \mathrm{mM}$ imidazole, prior to elution using either gradient elution or isocratic elution (dependent on the effector protein) with a buffer containing $50 \mathrm{mM}$ HEPES, pH 8.0, $300 \mathrm{mM} \mathrm{NaCl}$, and $250 \mathrm{mM}$ imidazole. Eluted fractions were analyzed by SDS-PAGE and fractions containing the protein of interest were dialyzed to remove imidazole and were incubated, with 6xHis-tagged 3C protease $(150 \mu \mathrm{g})$ overnight at $4^{\circ} \mathrm{C}$, to cleave off the $\mathrm{N}$-terminal fusion from the effector proteins. Cleavage was confirmed via SDS-PAGE, and the cleaved protein of interest was separated from the N-terminal fusion tag, any uncleaved protein, and 6xHis-tagged 3C protease using IMAC and were subsequently purified further by SEC, using either a HiLoad 16/600 or HiLoad 26/600 Superdex 75-pg column (Cytiva, Marlborough, MA, U.S.A.) equilibrated with a buffer containing $10 \mathrm{mM}$ HEPES, $\mathrm{pH} 8.0$, and $150 \mathrm{mM} \mathrm{NaCl}$. Proteins were concentrated using a 3- or 10-kDa molecular weight cut-off Amicon centrifugal concentrator (MilliporeSigma, Burlington, MA, U.S.A.), were snap-frozen in liquid nitrogen, and were stored at $-80^{\circ} \mathrm{C}$ for future use.

\section{Intact protein MS.}

Proteins were adjusted to $10 \mu \mathrm{M}$ in $0.1 \%$ (vol/vol) formic acid (FA) for high-pressure liquid chromatography (HPLC)-MS analysis. The samples were then injected onto an Agilent UHPLC system. Each sample was first desalted for $2 \mathrm{~min}$ on an Agilent (Santa Clara, CA, U.S.A.) C3 trap column (ZORBAX StableBond C3) at a flow rate of $500 \mu \mathrm{l} / \mathrm{min}$ at $95 \%$ buffer A $(0.1 \% \mathrm{FA}, \mathrm{vol} / \mathrm{vol})$ and $5 \%$ buffer B $(0.1 \% \mathrm{FA}$ and $100 \%$ acetonitrile) followed by separation over 8 min using a 5 to $80 \%$ ( $\mathrm{vol} / \mathrm{vol}$ ) gradient of buffer B at a flow rate of $500 \mu \mathrm{l} / \mathrm{min}$. Eluted material was analyzed using a Orbitrap Fusion Tribrid mass spectrometer (Thermo Fisher Scientific, Waltham, MA, U.S.A.). MS acquisition was performed using the Intact Protein Mode script. The acquisition was performed across $\mathrm{m} / \mathrm{z}, 200$ to 4,000 with an accumulation time of $1 \mathrm{~s}$. Data were analyzed using the Free Style v.1.4 (Thermo Fisher Scientific) protein reconstruct tool across a mass range of $\mathrm{m} / \mathrm{z}, 500$ to 2,000. The expected sizes of the proteins were searched.

\section{CD spectroscopy.}

The CD spectra of purified effectors of interest were recorded on a Chirascan spectrometer (Applied Photophysics Ltd., Leatherhead, U.K.) at $20^{\circ}$ C. Samples were diluted to $10 \mu \mathrm{M}$ in $20 \mathrm{mM}$ sodium phosphate buffer at $\mathrm{pH}$ 8.0. Measurements were taken at $0.5 \mathrm{~nm}$ wavelength increments from 190 or $200 \mathrm{~nm}$ to $260 \mathrm{~nm}$ at a scanning speed of $50 \mathrm{~nm} / \mathrm{min}$. A cell with a pathlength of $1 \mathrm{~mm}$, a bandwidth of $0.5 \mathrm{~nm}$, and response time of $4 \mathrm{~s}$ were used, with three accumulations. The data were averaged and corrected for buffer baseline contribution and were visualized using the webserver CAPITO tool with data smoothing (Wiedemann et al. 2013).

\section{Tomato infiltration assays.}

Tomato seeds were sown in seed-raising mix and were grown in a controlled environment chamber with a 16-h day and 8-h night cycle at $22^{\circ} \mathrm{C}$. Purified SIX4 (Avr1) protein was diluted in water to $0.1 \mathrm{mg} / \mathrm{ml}$. Syringe infiltrations of the cotyledons of 10-day-old tomato seedlings were conducted with $100 \mu \mathrm{l}$ of protein or buffer (10 mM HEPES, $\mathrm{pH} 8,150 \mathrm{mM} \mathrm{NaCl}$ diluted 1:100). Cotyledons were harvested and imaged at 4 days postinfiltration.

\section{ACKNOWLEDGMENTS}

We acknowledge T. Rhodes for access to modified cloning vectors and cloning advice. The vectors containing CyDisCo and FunCyDisCo were deposited with Addgene for easy access by the research community (Addgene ID numbers 176404 and 176405). The mass spectrometry analysis was carried out at the joint mass spectrometry facility at The Australian National University. We thank A. J. Carrol and J. Boileau for their technical assistance with the mass spectrometry experiments.

\section{LITERATURE CITED}

Almagro Armenteros, J. J., Tsirigos, K. D., Sønderby, C. K., Petersen, T. N., Winther, O., Brunak, S., von Heijne, G., and Nielsen, H. 2019 SignalP 5.0 improves signal peptide predictions using deep neural networks. Nat. Biotechnol. 37:420-423.

Bentham, A. R., Youles, M., Mendel, M. N., Varden, F. A., Concepcion, J. C. D. 1., and Banfield, M. J. 2021. pOPIN-GG: A resource for modular assembly in protein expression vectors. bioRxiv:2021.2008.2010. 455798.

Blondeau, K., Blaise, F., Graille, M., Kale, S. D., Linglin, J., Ollivier, B., Labarde, A., Lazar, N., Daverdin, G., Balesdent, M. H., Choi, D. H., Tyler, B. M., Rouxel, T., van Tilbeurgh, H., and Fudal, I. 2015. Crystal structure of the effector AvrLm4-7 of Leptosphaeria maculans reveals insights into its translocation into plant cells and recognition by resistance proteins. Plant J. 83:610-624.

De la Concepcion, J. C., Franceschetti, M., Maqbool, A., Saitoh, H., Terauchi, R., Kamoun, S., and Banfield, M. J. 2018. Polymorphic residues in rice NLRs expand binding and response to effectors of the blast pathogen. Nat. Plants 4:576-585.

Dodds, P. N., and Rathjen, J. P. 2010. Plant immunity: Towards an integrated view of plant-pathogen interactions. Nat. Rev. Genet. 11:539548.

Ellgaard, L., and Ruddock, L. W. 2005. The human protein disulphide isomerase family: Substrate interactions and functional properties. EMBO Rep. 6:28-32.

Frand, A. R., and Kaiser, C. A. 1999. Erolp oxidizes protein disulfide isomerase in a pathway for disulfide bond formation in the endoplasmic reticulum. Mol. Cell 4:469-477.

Gaciarz, A., Veijola, J., Uchida, Y., Saaranen, M. J., Wang, C., Hörkkö, S., and Ruddock, L. W. 2016. Systematic screening of soluble expression of antibody fragments in the cytoplasm of E. coli. Microb. Cell Fact. 15:22.

Iverson, S. V., Haddock, T. L., Beal, J., and Densmore, D. M. 2016 CIDAR MoClo: Improved MoClo assembly standard and new E. coli part library enable rapid combinatorial design for synthetic and traditional biology. ACS Synth. Biol. 5:99-103.

Lange, H., Lisowsky, T., Gerber, J., Mühlenhoff, U., Kispal, G., and Lill, R. 2001. An essential function of the mitochondrial sulfhydryl oxidase Erv1p/ALR in the maturation of cytosolic Fe/S proteins. EMBO Rep. 2:715-720.

Liu, Z., Faris, J. D., Oliver, R. P., Tan, K. C., Solomon, P. S., McDonald, M. C., McDonald, B. A., Nunez, A., Lu, S., Rasmussen, J. B., and Friesen, T. L. 2009. SnTox3 acts in effector triggered susceptibility to induce disease on wheat carrying the Snn3 gene. PLoS Pathog. 5: e1000581.

Liu, Z., Zhang, Z., Faris, J. D., Oliver, R. P., Syme, R., McDonald, M. C., McDonald, B. A., Solomon, P. S., Lu, S., Shelver, W. L., Xu, S., and Friesen, T. L. 2012. The cysteine rich necrotrophic effector SnTox 1 produced by Stagonospora nodorum triggers susceptibility of wheat lines harboring Snn1. PLoS Pathog. 8:e1002467.

Lobstein, J., Emrich, C. A., Jeans, C., Faulkner, M., Riggs, P., and Berkmen, M. 2012. SHuffle, a novel Escherichia coli protein expression strain capable of correctly folding disulfide bonded proteins in its cytoplasm. Microb. Cell Fact. 11:753.

Ma, L. J., van der Does, H. C., Borkovich, K. A., Coleman, J. J., Daboussi, M. J., Di Pietro, A., Dufresne, M., Freitag, M., Grabherr M., Henrissat, B., Houterman, P. M., Kang, S., Shim, W. B. Woloshuk, C., Xie, X., Xu, J. R., Antoniw, J., Baker, S. E., Bluhm, B. H., Breakspear, A., Brown, D. W., Butchko, R. A., Chapman, S., Coulson, R., Coutinho, P. M., Danchin, E. G., Diener, A., Gale, L. R., Gardiner, D. M., Goff, S., Hammond-Kosack, K. E., Hilburn, K., 
Hua-Van, A., Jonkers, W., Kazan, K., Kodira, C. D., Koehrsen, M., Kumar, L., Lee, Y. H., Li, L., Manners, J. M., Miranda-Saavedra, D., Mukherjee, M., Park, G., Park, J., Park, S. Y., Proctor, R. H., Regev, A., Ruiz-Roldan, M. C., Sain, D., Sakthikumar, S., Sykes, S., Schwartz, D. C., Turgeon, B. G., Wapinski, I., Yoder, O., Young, S., Zeng, Q., Zhou, S., Galagan, J., Cuomo, C. A., Kistler, H. C., and Rep, M. 2010. Comparative genomics reveals mobile pathogenicity chromosomes in Fusarium. Nature 464:367-373.

Maqbool, A., Saitoh, H., Franceschetti, M., Stevenson, C. E., Uemura, A., Kanzaki, H., Kamoun, S., Terauchi, R., and Banfield, M. J. 2015. Structural basis of pathogen recognition by an integrated HMA domain in a plant NLR immune receptor. eLife 4:e08709.

Marín-Menguiano, M., Moreno-Sánchez, I., Barrales, R. R., FernándezÁlvarez, A., and Ibeas, J. I. 2019. N-glycosylation of the protein disulfide isomerase Pdil ensures full Ustilago maydis virulence. PLoS Pathog. 15:e1007687.

Matos, C. F., Robinson, C., Alanen, H. I., Prus, P., Uchida, Y., Ruddock, L. W., Freedman, R. B., and Keshavarz-Moore, E. 2014. Efficient export of prefolded, disulfide-bonded recombinant proteins to the periplasm by the Tat pathway in Escherichia coli CyDisCo strains. Biotechnol. Prog. 30:281-290.

Moore, S. J., Lai, H. E., Kelwick, R. J., Chee, S. M., Bell, D. J., Polizzi, K. M., and Freemont, P. S. 2016. EcoFlex: A multifunctional MoClo kit for E. coli synthetic biology. ACS Synth. Biol. 5:1059-1069.

Nielsen, L. D., Foged, M. M., Albert, A., Bertelsen, A. B., Søltoft, C. L., Robinson, S. D., Petersen, S. V., Purcell, A. W., Olivera, B. M., Norton, R. S., Vasskog, T., Safavi-Hemami, H., Teilum, K., and Ellgaard, L. 2019. The three-dimensional structure of an H-superfamily conotoxin reveals a granulin fold arising from a common ICK cysteine framework. J. Biol. Chem. 294:8745-8759.

Nørgaard, P., Westphal, V., Tachibana, C., Alsøe, L., Holst, B., and Winther, J. R. 2001. Functional differences in yeast protein disulfide isomerases. J. Cell Biol. 152:553-562.

Oliveira-Garcia, E., and Valent, B. 2015. How eukaryotic filamentous pathogens evade plant recognition. Curr. Opin. Microbiol. 26:92-101.

Outram, M. A., Sung, Y. C., Yu, D., Dagvadorj, B., Rima, S. A., Jones, D. A., Ericsson, D. J., Sperschneider, J., Solomon, P. S., Kobe, B., and Williams, S. J. 2021. The crystal structure of SnTox3 from the necrotrophic fungus Parastagonospora nodorum reveals a unique effector fold and provides insight into Snn3 recognition and pro-domain protease processing of fungal effectors. New Phytol. 231:2282-2296.

Petre, B., and Kamoun, S. 2014. How do filamentous pathogens deliver effector proteins into plant cells? PLoS Biol. 12:e1001801.

Prahlad, J., Struble, L. R., Lutz, W. E., Wallin, S. A., Khurana, S., Schnaubelt, A., Broadhurst, M. J., Bayles, K. W., and Borgstahl, G. E. O. 2021. CyDisCo production of functional recombinant SARSCoV-2 spike receptor binding domain. Protein Sci. 30:1983-1990.

Sánchez-Vallet, A., Saleem-Batcha, R., Kombrink, A., Hansen, G., Valkenburg, D. J., Thomma, B. P., and Mesters, J. R. 2013. Fungal effector Ecp6 outcompetes host immune receptor for chitin binding through intrachain LysM dimerization. eLife 2:e00790.

See, P. T., Iagallo, E. M., Oliver, R. P., and Moffat, C. S. 2019. Heterologous expression of the Pyrenophora tritici-repentis effector proteins ToxA and ToxB, and the prevalence of effector sensitivity in Australian cereal crops. Front. Microbiol. 10:182.

Selin, C., de Kievit, T. R., Belmonte, M. F., and Fernando, W. G. 2016. Elucidating the role of effectors in plant-fungal interactions: Progress and challenges. Front. Microbiol. 7:600.

Sevier, C. S. 2010. New insights into oxidative folding. J. Cell Biol. 188: 757-758.

Sevier, C. S., Cuozzo, J. W., Vala, A., Aslund, F., and Kaiser, C. A. 2001. A flavoprotein oxidase defines a new endoplasmic reticulum pathway for biosynthetic disulphide bond formation. Nat. Cell Biol. 3: 874-882.

Sevier, C. S., and Kaiser, C. A. 2006. Conservation and diversity of the cellular disulfide bond formation pathways. Antioxid. Redox Signal. 8: 797-811.

Sohail, A. A., Gaikwad, M., Khadka, P., Saaranen, M. J., and Ruddock, L. W. 2020. Production of extracellular matrix proteins in the cytoplasm of E. coli: Making giants in tiny factories. Int. J. Mol. Sci. 21: 688.

Stergiopoulos, I., and de Wit, P. J. 2009. Fungal effector proteins. Annu. Rev. Phytopathol. 47:233-263.

Sung, Y. C., Outram, M. A., Breen, S., Wang, C., Dagvadorj, B., Winterberg, B., Kobe, B., Williams, S. J., and Solomon, P. S. 2021 PR1-mediated defence via C-terminal peptide release is targeted by a fungal pathogen effector. New Phytol. 229:3467-3480.

Widmann, M., and Christen, P. 2000. Comparison of folding rates of homologous prokaryotic and eukaryotic proteins. J. Biol. Chem. 275: 18619-18622.

Wiedemann, C., Bellstedt, P., and Görlach, M. 2013. CAPITO—A web server-based analysis and plotting tool for circular dichroism data. Bioinformatics 29:1750-1757.

Wilson, R. H., Thieulin-Pardo, G., Hartl, F. U., and Hayer-Hartl, M. 2019. Improved recombinant expression and purification of functional plant Rubisco. FEBS Lett. 593:611-621.

Yi, M., Chi, M. H., Khang, C. H., Park, S. Y., Kang, S., Valent, B., and Lee, Y. H. 2009. The ER chaperone LHS1 is involved in asexual development and rice infection by the blast fungus Magnaporthe oryzae. Plant Cell 21:681-695.

Zhang, X., Nguyen, N., Breen, S., Outram, M. A., Dodds, P. N., Kobe, B., Solomon, P. S., and Williams, S. J. 2017. Production of small cysteine-rich effector proteins in Escherichia coli for structural and functional studies. Mol. Plant Pathol. 18:141-151.

Zhou, Y., Lu, Z., Wang, X., Selvaraj, J. N., and Zhang, G. 2018. Genetic engineering modification and fermentation optimization for extracellular production of recombinant proteins using Escherichia coli. Appl. Microbiol. Biotechnol. 102:1545-1556. 